\title{
Semantic annotation of biological concepts interplaying microbial cellular responses
}

\author{
Rafael Carreira ${ }^{1}{ }^{2}$, Sónia Carneiro ${ }^{1}$, Rui Pereira ${ }^{1}$, Miguel Rocha ${ }^{2}$, Isabel Rocha ${ }^{1}$, Eugénio C Ferreira ${ }^{1}$ and \\ Anália Lourenço ${ }^{1 *}$
}

\begin{abstract}
Background: Automated extraction systems have become a time saving necessity in Systems Biology. Considerable human effort is needed to model, analyse and simulate biological networks. Thus, one of the challenges posed to Biomedical Text Mining tools is that of learning to recognise a wide variety of biological concepts with different functional roles to assist in these processes.
\end{abstract}

Results: Here, we present a novel corpus concerning the integrated cellular responses to nutrient starvation in the model-organism Escherichia coli. Our corpus is a unique resource in that it annotates biomedical concepts that play a functional role in expression, regulation and metabolism. Namely, it includes annotations for genetic information carriers (genes and DNA, RNA molecules), proteins (transcription factors, enzymes and transporters), small metabolites, physiological states and laboratory techniques. The corpus consists of 130 full-text papers with a total of 59043 annotations for 3649 different biomedical concepts; the two dominant classes are genes (highest number of unique concepts) and compounds (most frequently annotated concepts), whereas other important cellular concepts such as proteins account for no more than $10 \%$ of the annotated concepts.

Conclusions: To the best of our knowledge, a corpus that details such a wide range of biological concepts has never been presented to the text mining community. The inter-annotator agreement statistics provide evidence of the importance of a consolidated background when dealing with such complex descriptions, the ambiguities naturally arising from the terminology and their impact for modelling purposes.

Availability is granted for the full-text corpora of 130 freely accessible documents, the annotation scheme and the annotation guidelines. Also, we include a corpus of 340 abstracts.

\section{Background}

Due to its latest achievements, text mining, i.e. the automated extraction of information from electronically published sources, is receiving increasing interest from the scientific community [1-4]. Text mining has been accounted for in the literature curation pipelines of several databases devoted to cellular modelling, namely: Regulon's network of transcriptional regulation in Escherichia coli (E. coli) [5], the Open Regulatory Annotation database (ORegAnno) on cis-regulatory data [6], the KInetic Database (KID) [7] and the BRaunschweig ENzyme Database (BRENDA) [8] both covering kinetic enzyme information,

\footnotetext{
*Correspondence: analia@deb.uminho.pt

${ }^{1}$ IBB - Institute for Biotechnology and Bioengineering, Centre of Biological Engineering, University of Minho, Campus de Gualtar, 4710-057 Braga PORTUGAL

Full list of author information is available at the end of the article
}

the Mouse Genome Informatics (MGI) database [9], the STRING protein-protein interaction database [10], and a knowledge base on molecular mechanisms of bacterial enteropathogens [11]. Moreover, development efforts have been made to provide tools able to combine text mining techniques and manual curation into customised modelling workflows [4,12-14].

Here, we use our expertise on modelling microbial cellular processes to present a new set of annotated resources, addressing key modelling necessities, to be used by the text mining community. We provide corpora concerning the integrated cellular responses to nutrient starvation in the model-organism $E$. coli, in which a variety of metabolic and regulatory biological concepts with assorted functional roles is identified. Our annotation guidelines and our evaluation of Inter-Annotator Agreement (IAA) address biological issues such as: the specification of the biological
C Biomed Central

() 2011 Carreira et al; licensee BioMed Central Ltd. This is an Open Access article distributed under the terms of the Creative Commons Attribution License (http://creativecommons.org/licenses/by/2.0), which permits unrestricted use, distribution, and reproduction in any medium, provided the original work is properly cited. 
concepts most relevant for studying cellular systems, how their basic functional roles can be fitted into a taxonomy of nominal classes and the terminological ambiguities that are likely to occur on microbial-related literature. Also, we analyse the impact of having annotators with different (levels of) expertise involved in such a process and describe the refinement of our annotation guidelines according to the outputs of annotator training.

The most relevant contents are available in supplementary material, namely: the corpus (available in multiple formats), data about the training stage (e.g. reports and guidelines), all IAA calculations and some other data considered useful to the community.

\section{Related work}

Semantically annotated corpora are commonly used to train algorithms to extract information considered of interest. However, the construction of a corpus tends to be a laborious and time-consuming task that requires considerable domain expertise to guarantee the highquality (correctness and meaningfulness) of the annotations. Evaluation efforts, such as BioCreative $[15,16]$, TREC $[17,18]$ and BioNLP [19], as well as individual text mining research projects, have tailored a number of corpora, freely available for the community.

Apart from their domain-specificities, corpora can be distinguished on the basis of the text segments included and the diversity of concepts involved. For a while, publishing policies and the costs associated with manual annotation dictated the use of abstracts or smaller segments (such as a randomised set of sentences) to construct corpora [20-25]. Although such segments hold a limited amount of information [26], this fact did not represent an actual research constraint as much had to be done in terms of training algorithms and building models. After all, state-of-the-art recognisers for key entities like genes, proteins and compounds are no more than five years old [27-31]. Meanwhile, the urge to develop fully equipped information extraction systems is demanding the construction and use of corpora of full-text documents [32-34], as well as the annotation of relationships/events [24,35-37].

Although the new corpora are of interest to Systems Biology applications and tools, most of them do not yet cover the desired holistic annotation of cellular processes. Except for a few recent works $[24,35]$, existing corpora cover for a limited set of biological concepts (often, just genes and proteins) and annotation tends to focus on particular cellular processes (e.g. protein-protein interactions or transcriptional regulation), rather than to address integrated cellular processes (e.g. effects of transcriptional regulation over enzymatic reactions).

\section{Motivation and objectives}

When aiding in the construction of cellular models, text mining systems have to deal with a wide variety of biological concepts with different functional roles. Information on gene expression and metabolic activities are at the core of cellular growth, development, reproduction and adaptability to environmental changes. Therefore, publications in the field often include the description of complex interactions involving genes, regulatory proteins (transcriptions factors and sigma factors), enzymes (and/or the catalytic reactions triggered by them), and metabolites (small molecules or compounds). Until now, a corpus that details a wide range of biological concepts has never been presented to the text mining community, thereby limiting the application of text mining on modelling tools. Particularly, one cannot forget that model reconstruction depends extensively on literature curation rather than the contents of databases [38] and thus, any means to enhance curation and integrate text mining facilities in modelling tools are of great interest to the community.

In this paper, we explore the issues that text mining systems will need to face in order to handle the knowledge contained within full-text articles. Since cellular modelling is one of our main research lines [39-43] and we have been evaluating text mining approaches in previous work $[13,44]$, we are in a privileged position to provide both biologists' and modellers' perspectives about the construction of text mining resources. In particular, we investigate domain-specific semantic issues, namely ambiguities arising from terminology (e.g. polysemy and synonymy) and biological concepts playing multiple functional roles, by focussing on the manual identification of a set of key entities to cellular modelling within full-text articles. As final outcome, we provide two annotated corpora: a corpus of 130 full-text documents and a corpus of 340 abstracts.

In the remainder of this paper, we firstly introduce the key aspects of the annotation scheme, the annotation software used and the profiles and training of the annotators. Subsequently, we detail the construction of our corpus of full-text documents, quantifying and explaining annotation discrepancies and describing the post-processing that ensures the quality of the final corpus. Then, we perform a comparative analysis of our corpus of full-text documents and our corpus of abstracts regarding the number and diversity of biological concepts that have been annotated. Some conclusions and future work directions are stated at the end.

\section{Methods}

This section characterises our corpora and describes the preparatory work required prior to their annotation. Since the preparatory work, annotation and analysis of our corpus of full-texts and our corpus of abstracts have 
been performed in a similar way, we describe the process applied to the corpus of full-texts, while the data corresponding to the corpus of abstracts is provided in supplementary material.

\section{Corpora characterisation}

The candidate documents for our work were retrieved from PubMed, using the keywords ("Escherichia coli" and "stringent response"), in January 2010. These candidate documents were screened for relevance by two of the authors with biological expertise, resulting in a set of 340 documents. From this set, we constructed a corpus of 340 abstracts and a corpus of 130 open-access full-texts.

The process of annotation of the two corpora was the same. Specifically, annotators followed the same annotation scheme and guidelines, the quality of their annotations was quantified similarly and identical post-processing ensured the high-quality of the final annotations. The effort expended by the two annotators amounted to a total of approximately 1188 person hours (equivalent to 6.75 person months).

The corpora are delivered in two XML-based formats: inline annotation and stand-off annotation. Individual annotations comprise the text span and its offsets (referring to the full extent of the text), a category from our hierarchy of biological concept types (see Annotation scheme section), and, whenever possible, the database identifier (i.e. an identifier from EcoCyc [45] or PSI-MI [46]) and the associated common name. In supplementary material we provide the list of PubMed identifiers (PMIDs) of the documents that compose our corpora (additional file "List of Documents") as well as the annotated corpus of full-text documents (additional files "FulltextsCorpus_Inline" and "FulltextsCorpus_Standoff") and the annotated corpus of abstracts (additional files "AbstractsCorpus_Inline" and "AbstractsCorpus_Standoff").

\section{Annotation scheme}

We have arranged biological concepts into the following semantic categories (Figure 1): genetic information carrier (which includes the categories gene, dna and rna), protein (which includes the categories enzyme and transcription factor), compound, biochemical reaction, physiological state and laboratory technique. Besides common biological concepts, we are interested in growth and environmental conditions or changes, i.e. conditions that somehow trigger regulatory actions at either the transcriptional or the metabolic level (e.g. cell adaptation to osmotic variations or nutrient deprivation). We are also interested in the laboratory techniques used to identify biological entities (e.g. mass spectrometry) and characterise the underlying properties of the biological systems involving these entities (e.g. in vitro enzymatic assays).

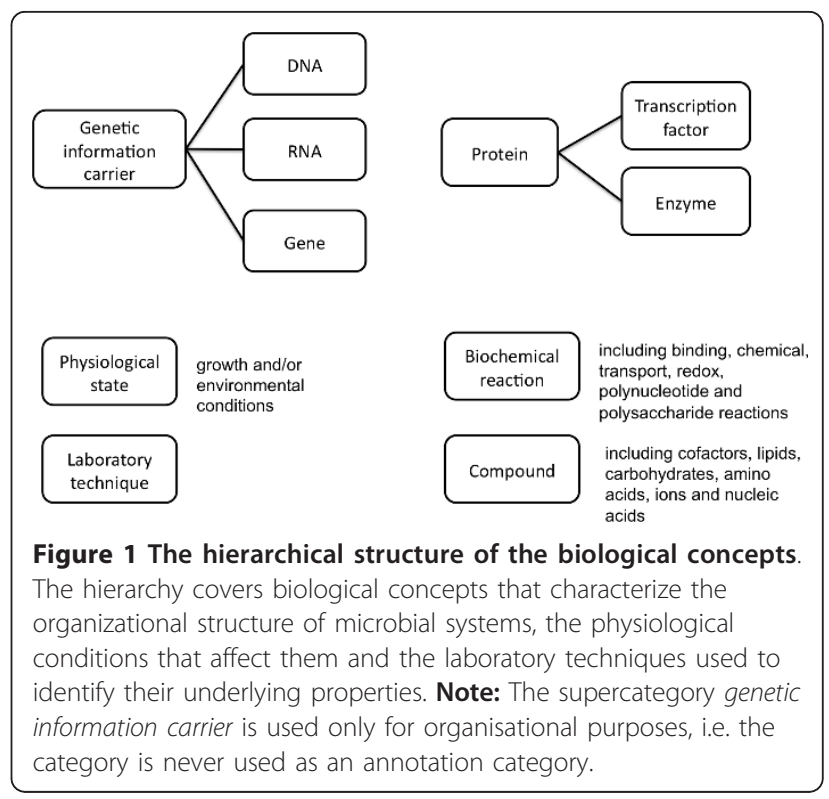

Each biological concept has a number of possible names associated (i.e. all the text forms that annotators have recognised in the documents), but has unambiguous meaning and can be associated with a unique database identifier. Namely, we have used the EcoCyc knowledge base, a key resource for $E$. coli studies, to organise molecular information and the Molecular Interactions (PSI-MI) ontology to index laboratory techniques.

To attain high quality annotations, we have produced a detailed set of annotation guidelines that describe the biological concepts pertaining to each semantic category, and provide clear positive and negative examples of concept annotation. The structure and content of these guidelines were iteratively refined in discussion with domain experts and annotators, via group discussions after the training cycles and after the post-processing of the corpora. The final guidelines of annotation can be found in additional file "Final Guidelines" in supplementary material.

\section{Annotation software}

The retrieval of documents and the subsequent manual annotation was performed using @Note, a workbench for Biomedical Text Mining [47]. This workbench enables keyword-based PubMed querying and document retrieval. Moreover, it provides a user-friendly interface for document visualisation and annotation: a colour-based scheme keeps visual track of any annotation editing, whilst another panel shows updated statistics on document and corpus annotation; a browsing mechanism allows the annotator to query well-known databases about name variants; and, a basic statistics feature characterises the ongoing annotation in terms of the number of concepts and associated annotations for each category. 
See additional file "Software Snapshots" in supplementary material for illustrative snapshots of the process.

\section{Annotator profiling}

The entanglement of biological concepts in the description of cellular processes is not straightforward to follow by those who do not have enough familiarity with biochemical pathways and regulatory cascades. Therefore, although the availability of annotators is often quite limited, it is important to establish the minimum level of expertise to be required for the production of such a corpus and the level of detail that annotation guidelines have to encompass in order to ensure the quality of annotation.

To perform this exercise, and given the availability of human resources, we chose two annotators with different levels of expertise. A student finishing a $\mathrm{PhD}$ thesis on the stringent response of $E$. coli (designated as annotator A) and a student who has recently started his $\mathrm{PhD}$ in $E$. coli bioprocesses (designated as annotator B). Both annotators present near-native competence in English and have solid background in Biology, but one differs from the other in terms of familiarity with microbial cellular processes and the E. coli stress phenomena.

\section{Annotator training and quality control}

Our training process included a preliminary session and three training cycles. First, we introduced the annotation tool and the task, exemplifying the annotation guidelines with some positive and negative examples of the biological concepts to be annotated (see additional file "Initial Guidelines" in supplementary material). Next, we selected a subset of 45 documents to be presented to both annotators and initiated the training cycles. Each training cycle included the annotation of the corpus based on the last updates on the guidelines (see additional files "Report after cycle 1" and "Report after cycle 2" in supplementary material), the calculation of the rates of agreement between annotators, the examination of the discrepancies and the refinement of the guidelines.

To quantify the improvement in the rates of agreement, we calculated the F-score, a common metric in IAA evaluations $[24,28,35]$.

$$
\begin{aligned}
& F-\text { score }=2 \times \frac{\text { precison } \times \text { recall }}{\text { precision }+ \text { recall }} \\
& \text { Precision }=\frac{\text { number of identical entities in set } A \text { and set } B}{\text { number of entities in set } A} \\
& \text { Recall }=\frac{\text { number of identical entities in set } A \text { and set } B}{\text { number of entities in set } B}
\end{aligned}
$$

Legend: Set A refers to the set of annotations produced by annotator A and set B refers to the set of annotations produced by annotator $\mathrm{B}$. Also, note that $\operatorname{recall}($ set $A$, set $B)=\operatorname{precision}($ set $B$, set $A)$ [48].

Then, two of the authors (with extensive biological expertise in the field) examined the annotation discrepancies and, prior to the next cycle of annotation, the group discussed the observed ambiguities/glitches and revised the annotation guidelines accordingly (e.g. by introducing more positive and negative examples of the different concepts).

In general, the trend was for the rates of agreement to improve between training cycles (Table 1). At the end of the last cycle, most agreement levels were approximate to or above $50 \%$, which we consider to be acceptable regarding the different levels of expertise of our annotators and the expected complexity in assigning some of the concepts.

Indeed, we concluded that the slight decrease of the rates of agreement for protein and transcription factor in the last cycle is caused by different levels of experience on gene regulation and also, the distinction between biochemical reaction and enzyme is unclear to less experienced annotators.

\section{Results and discussion}

After the training period, the corpus of 130 full-texts and the corpus of 340 abstracts were constructed (all documents have been annotated by both annotators following the same collection of rules detailed in additional file "Final Guidelines" in supplementary material).

In this section, we detail the process of annotation for the corpus of 130 full-texts, examining the IAA scores

Table 1 Inter-annotator agreement during training

\begin{tabular}{cccc}
\hline & Training cycle & Training cycle & Training cycle \\
& $\mathbf{1}$ & $\mathbf{2}$ & $\mathbf{3}$ \\
\hline dna & - & $5.74 \%$ & $21.74 \%$ \\
\hline rna & - & $55.81 \%$ & $65.63 \%$ \\
\hline gene & $69.39 \%$ & $63.26 \%$ & $83.08 \%$ \\
\hline protein & - & $52.71 \%$ & $48.45 \%$ \\
\hline enzyme & $41.03 \%$ & $53.78 \%$ & $65.28 \%$ \\
\hline $\begin{array}{c}\text { transcription factor } \\
\text { compound }\end{array}$ & $0 \%$ & $38.46 \%$ & $20.51 \%$ \\
\hline $\begin{array}{c}\text { biochemical } \\
\text { reaction }\end{array}$ & $05.28 \%$ & $65.36 \%$ & $71.54 \%$ \\
\hline $\begin{array}{c}\text { physiological state } \\
\text { laboratory } \\
\text { technique }\end{array}$ & $27.85 \%$ & $0 \%$ & $0 \%$ \\
\hline Coluns report the & $23.01 \%$ & $40.94 \%$ & $42.51 \%$ \\
\hline
\end{tabular}

Columns report the F-scores obtained for each semantic category after a cycle of training. Note that the annotation of concepts for categories dna and rna (under the supercategory genetic information carrier, which also includes the category gene) and protein (that aggregates the subcategories enzyme and transcription factor) was considered only after the first training cycle. 
obtained and explaining the post-processing that was executed to reach a biologically consistent consensus and eliminate annotation inconsistencies. Further on, we provide the general statistics of the final corpora and perform a comparison regarding the distribution of concepts per semantic category.

\section{Quality control}

Since, during the training period, we concluded that part of the annotation inconsistencies were due to the different levels of expertise of the annotators, we were expecting similar problems at the annotation of the final corpus (Table 2). Indeed, although the rates of agreement for most categories are fairly good (approximately or above $50 \%$ ), the only category that achieves high agreement is gene, while categories such as transcription factor and dna (about concepts related to regulatory functions) present lower scores.

Thus, we decided to explore the nature of the discrepancies by detailing the assignments of the annotators per semantic category (Table 3). Specifically, we had a round of group meetings where we examined discrepancies in category assignment, assignments to fine-grained categories and differences in the number of annotations.

\section{Polysemy}

Situations of polysemy (i.e. when a name, one or more words, may be used to denote different biological concepts belonging to different semantic categories) caused many category disagreements. Namely, we observe this problem between the categories gene and compound, gene and protein, protein and compound, and enzyme and biochemical reaction.

For example: names such as "leu", "mal" and "fum" are names of genes but are also acronyms of the compounds "leucine", "maltose" and "fumarate"; in the domain of our corpus, the name "stringent factor" is a synonym for both the compound "ppGpp" and the enzyme "RelA"; the name "gpp" is a synonym for a gene coding for the enzymes

Table 2 Inter-annotator agreement for the 130 full-texts

\begin{tabular}{cc}
\hline & Final F-score \\
\hline dna & $13.22 \%$ \\
\hline rna & $59.69 \%$ \\
\hline gene & $91.78 \%$ \\
\hline protein & $42.15 \%$ \\
\hline enzyme & $63.33 \%$ \\
\hline transcription factor & $28.13 \%$ \\
\hline compound & $63.90 \%$ \\
\hline biochemical reaction & $0 \%$ \\
\hline physiological state & $46.50 \%$ \\
\hline laboratory technique & $38.34 \%$ \\
\hline
\end{tabular}

F-scores were estimated for each semantic category. "guanosine-5'-triphosphate, 3'-diphosphate pyrophosphatase" and "xanthine-guanine phosphoribosyltransferase" and the compound "geranyl diphosphate"; and the name "glyD" is a synonym for a geneand an enzyme. Also, the names of reactions are easily misinterpreted with the names of enzymes, because the latest are mainly derived from the designation of the respective catalysing biochemical reaction ending in 'ase' and its substrate name (e.g. sulfate adenylyltransferase catalyses the transfer of phosphorus-containing nucleotide groups to sulphate (substrate), i.e. performs the adenylyltransferase activity).

\section{Synonyms/name variants}

The high degree of synonymy found in biological vocabularies represents a challenge to the comprehensiveness of the annotation. Indeed, the use of different names to denote the same biological concept requires a certain familiarity with the field, as many synonyms are not catalogued in databases and many name variants are only associated with a biological concept in a very specific context. Just to exemplify, during the annotation of our corpus, we have found 27 name variants of the compound "ppGpp", 23 name variants of the enzyme "RelA", and 5 name variants for the transcription factor "CRP-cAMP".

\section{Fine-grained semantics}

Annotator B assigned many transcription factor and enzyme concepts to protein. For example, non-obvious enzyme names (e.g. "penicillin binding protein 2", and the synonyms "OmpT" and "outer membrane protein $3 \mathrm{~b}$ ") and transcription factors (e.g. "ntrc" that designates the $\mathrm{NtrC}$ transcriptional dual regulator and "ole" that designates the FadR DNA-binding transcriptional dual regulator) were annotated as protein.

\section{Misclassification of biological concepts}

Some category disagreements were seen to be due to misclassifications by one of the annotators. The reasons behind these misclassifications are somewhat difficult to unravel, but it could be attributed to misinterpretations in specific biological contexts. For example, we found out that annotator B misclassified some compounds as dna (e. g. "mal" that is an abbreviation of "maltose", a compound that is used as a carbon source by many organisms) or as enzyme (e.g. "luciferin" which is a class of small-molecules that are oxidized in the presence of the enzyme luciferase to produce oxyluciferin and energy in the form of light). Annotator $\mathbf{B}$ also annotated most transcription units (e.g. "thrAC", "tauABCD", and "sucACD") and operons (e.g. "gltB operon" and " ftsQAZ operon") as gene and misclassified some laboratory techniques(e.g. "sonication" as physiological state, "autoradiography" as compound and "dideoxysequencing reaction" as biochemical reaction).

\section{Exclusively annotated concepts}

The extent of biological concepts that were exclusively annotated by each of the annotators is large and fall particularly into categories like compound, laboratory 
Table 3 Annotator assignments per category

\begin{tabular}{|c|c|c|c|c|c|c|c|c|c|c|c|c|}
\hline & & \multicolumn{9}{|c|}{ Annotator B } & \multirow[b]{2}{*}{ physiological state } & \multirow[b]{2}{*}{ None } \\
\hline & & $d n a$ & $r n a$ & gene & protein & enzyme & transcription factor & compound & biochemical reaction & laboratory technique & & \\
\hline \multirow[t]{11}{*}{ Annotator A } & $d n a$ & $\begin{array}{c}8 \\
(2316)\end{array}$ & 0 & 0 & 0 & 0 & 0 & 0 & 0 & 0 & 0 & $\begin{array}{c}58 \\
(204)\end{array}$ \\
\hline & $r n a$ & 0 & $\begin{array}{c}57 \\
(9078)\end{array}$ & 0 & 0 & 0 & 0 & 0 & 0 & 0 & $\begin{array}{c}1 \\
(64)\end{array}$ & $\begin{array}{c}23 \\
(432)\end{array}$ \\
\hline & gene & 0 & 0 & $\begin{array}{c}1066 \\
(16250)\end{array}$ & $\begin{array}{c}3 \\
(68)\end{array}$ & 0 & 0 & $\begin{array}{c}3 \\
(534)\end{array}$ & 0 & 0 & 0 & $\begin{array}{c}59 \\
(276)\end{array}$ \\
\hline & protein & 0 & 0 & $\begin{array}{c}4 \\
(564)\end{array}$ & $\begin{array}{c}55 \\
(3396)\end{array}$ & $\begin{array}{c}5 \\
(18)\end{array}$ & 0 & $\begin{array}{c}2 \\
(64)\end{array}$ & 0 & 0 & 0 & $\begin{array}{c}53 \\
(261)\end{array}$ \\
\hline & enzyme & 0 & 0 & 0 & $\begin{array}{c}3 \\
(12)\end{array}$ & $\begin{array}{c}196 \\
(5457) \\
\end{array}$ & 0 & 0 & 0 & 0 & 0 & $\begin{array}{c}52 \\
(219) \\
\end{array}$ \\
\hline & transcription factor & 0 & $\begin{array}{c}1 \\
(20)\end{array}$ & $\begin{array}{l}2 \\
(6) \\
\end{array}$ & $\begin{array}{c}7 \\
(1510) \\
\end{array}$ & 0 & $\begin{array}{c}9 \\
(231)\end{array}$ & 0 & 0 & 0 & 0 & $\begin{array}{c}27 \\
(431) \\
\end{array}$ \\
\hline & compound & $\begin{array}{c}3 \\
(170)\end{array}$ & 0 & $\begin{array}{c}1 \\
(8)\end{array}$ & 0 & $\begin{array}{c}1 \\
(68)\end{array}$ & 0 & $\begin{array}{c}369 \\
(36857)\end{array}$ & 0 & 0 & 0 & $\begin{array}{c}75 \\
(683) \\
\end{array}$ \\
\hline & biochemical reaction & 0 & 0 & 0 & 0 & $\begin{array}{c}48 \\
(1425) \\
\end{array}$ & 0 & 0 & 0 & 0 & 0 & $\begin{array}{c}13 \\
(48) \\
\end{array}$ \\
\hline & laboratory technique & 0 & 0 & 0 & $\begin{array}{c}1 \\
(18)\end{array}$ & $\begin{array}{c}1 \\
(6)\end{array}$ & 0 & $\begin{array}{c}2 \\
(183)\end{array}$ & 0 & $\begin{array}{c}136 \\
(3495)\end{array}$ & $\begin{array}{c}1 \\
(40)\end{array}$ & $\begin{array}{c}121 \\
(852)\end{array}$ \\
\hline & physiological state & 0 & 0 & 0 & 0 & 0 & 0 & 0 & 0 & 0 & $\begin{array}{c}97 \\
(8361) \\
\end{array}$ & $\begin{array}{c}210 \\
(5397) \\
\end{array}$ \\
\hline & None & $\begin{array}{c}44 \\
(1555)\end{array}$ & $\begin{array}{c}52 \\
(130)\end{array}$ & $\begin{array}{c}119 \\
(529)\end{array}$ & $\begin{array}{c}73 \\
(440)\end{array}$ & $\begin{array}{c}117 \\
(941)\end{array}$ & $\begin{array}{c}9 \\
(30)\end{array}$ & $\begin{array}{c}330 \\
(2163)\end{array}$ & $\begin{array}{c}1 \\
(1)\end{array}$ & $\begin{array}{c}187 \\
(575)\end{array}$ & $\begin{array}{c}100 \\
(786)\end{array}$ & \\
\hline
\end{tabular}

Cells represent the assignments of both annotators in terms of the number of biological concepts and the corresponding number of annotations (depicted between parentheses). Consensual assignments, i.e.
assignments to the same category, are depicted at the diagonal of the table in bold; discrepancies in category assignment are indicated by non-diagonal cells; and the pseudo-category "None" represents all assignments made by only one of the annotators. For instance, the top left hand cell indicates that annotators agreed on 8 biological concepts for the category dna, corresponding to a total of 2316 annotations. 
technique and physiological state. Annotator $\mathbf{B}$ tended to annotate more exclusive terms than annotator $\mathbf{A}$, in particular in gene category (119 and 59 exclusive terms, respectively), enzyme category (117 and 52 exclusive terms, respectively) and compound category (330 and 75 exclusive terms, respectively). It was observed that Annotator B did not strictly follow some of the guidelines and annotated genes that do not belong to E. coli or were introduced via genetic transformations (e.g. the gene bmpA is from the bacteria Borrelia burgdorferi), or terms that should be assigned to the biochemical reaction in the enzyme category (e.g. hydrolase is not an enzyme but the activity performed by several enzymes) and also, compounds that do not participate in biochemical reactions within $E$. coli cells, but are used in laboratory assays (e.g. azthreonam that is a synthetic monocyclic beta-lactam antimicrobial agent).

In categories like laboratory technique and physiological state, the number of concepts exclusively annotated was roughly even for both annotators. Apparently, the annotation of these biological concepts is highly dependent on the background knowledge of the annotators. As observed, annotator A annotated many more concepts in the physiological state category. This can be explained by the fact that he is more familiar with the case study (i.e. the stringent response in E. coli) compared to annotator B. Likewise, the extent of exclusive terms assigned to the laboratory technique category was conditioned by the experience of each annotator. While annotator $\mathbf{B}$ has considered more laboratory techniques related to biochemistry and genetics (e.g. reverse transcriptase mapping or ribonuclease protection assays), annotator A annotated more techniques that are related to preparative or analytical techniques for measuring analytes (e.g. chip immunoprecipitation or capillary electrophoresis).

\section{Post-processing}

After identifying the main issues affecting annotation consistency, we undertook a final round of group sessions to resolve them. Most inconsistencies were resolved in favour of the more experienced annotator, specifically inconsistencies related to the above mentioned fine-grained category assignments, exclusively annotated concepts, and polysemy and synonymy situations.

To guarantee the high-quality of the final corpus we have revised the annotations as follows:

- biochemical reaction: since the disagreements throughout the whole process of training and corpus annotation were considerable, it was decided to not include this biological concept in the final corpus. Therefore, in addition to the previous rules regarding the transition of terms between the enzyme and biochemical reaction concepts, it was determined to eliminate this biological concept from the final corpus.

- compound: the level of exclusive terms included in the 130 full-text corpus by the Annotator $\mathbf{B}$ is considerably higher when compared to those annotated by Annotator A. The annotation of compounds that are uniquely used in biochemical assays (e.g. EDTA or acetonitrile) was previously decided (see additional file "Report after cycle 1") to be disregarded. However, some of these terms were still assigned as compounds and, consequently, were filtered out;

- gene: similarly to what was seen in compound annotations, the addition of genes that are coming from other biological sources other than $E$. coli must be disregarded. Thus, it was agreed that any term that refers to a gene from another organism will be omitted (e.g. bmpA, bmpB or bmpD designate genes from Borrelia burgdorferi and should not be annotated).

- laboratory technique: the number of exclusive terms annotated by both annotators exceeds the number of concepts in which annotators agreed. This denotes the importance of the annotator expertise at specific research areas as explained before. In this case, it was decided to join annotations from both annotators;

- physiological state: annotations on this category were also disparate when analysing exclusive terms from both annotators and were most likely a consequence of the level of expertise of the two annotators. Since annotator A was more familiar with the case study (i.e. the stringent response in E. coli) many more terms were classified. Nevertheless, annotator B assigned some terms that were missed by annotator $\mathbf{A}$ and it was decided to include them in the final corpus.

\section{Final corpus statistics}

After ensuring the consistency of the annotations, our corpus of 130 full-text documents comprises 59043 annotations, corresponding to 3649 unique biological concepts, distributed according to the categories of our scheme as shown in Table 4.

Gene and compound are the categories with the largest number of biological concepts annotated. This is explained by the fact that most activities related to metabolism and gene expression are described to some extent by concepts of these two categories. Regarding the ratio of biological concepts, the category compound is about $10 \%$ below the category gene, but its ratio of annotations is almost $20 \%$ higher than the category gene. This means that we have annotated more gene concepts but comparatively, the documents contain more mentions of compound concepts. Once again, this is explained by the fact that $E$. coli stringent response is triggered by compounds, (p)ppGpp, and then influences 
Table 4 General statistics about the corpus of full-text documents

\begin{tabular}{|c|c|c|c|c|c|c|c|}
\hline \multicolumn{2}{|c|}{ Categories } & \multirow{2}{*}{$\begin{array}{c}\text { \#concepts } \\
126\end{array}$} & \multirow{2}{*}{$\begin{array}{c}\begin{array}{c}\# \\
\text { annotations }\end{array} \\
3771\end{array}$} & \multirow{2}{*}{$\begin{array}{c}\begin{array}{c}\% \\
\text { concepts }\end{array} \\
3.45 \% \\
\end{array}$} & \multirow{2}{*}{$\begin{array}{c}\begin{array}{c}\% \\
\text { annotations }\end{array} \\
6.39 \%\end{array}$} & \multirow{2}{*}{$\begin{array}{c}\begin{array}{c}\text { Annotation } \\
\text { Frequency }\end{array} \\
29.93\end{array}$} & \multirow{2}{*}{$\begin{array}{c}\begin{array}{c}\text { Concept } \\
\text { Distribution }\end{array} \\
8.87\end{array}$} \\
\hline Genetic Information & $d n a$ & & & & & & \\
\hline & rna & 119 & 3970 & $3.26 \%$ & $6.72 \%$ & 33.36 & 8.38 \\
\hline & gene & 1175 & 8770 & $32.20 \%$ & $14.85 \%$ & 7.46 & 82.75 \\
\hline \multirow[t]{3}{*}{ Protein } & protein & 175 & 2332 & $4.80 \%$ & $3.95 \%$ & 13.33 & 28.69 \\
\hline & enzyme & 388 & 4025 & $10.63 \%$ & $6.82 \%$ & 10.37 & 63.61 \\
\hline & $\begin{array}{l}\text { transcription } \\
\text { factor }\end{array}$ & 47 & 1434 & $1.29 \%$ & $2.43 \%$ & 30.51 & 7.70 \\
\hline \multicolumn{2}{|c|}{ compound } & 767 & 21414 & $21.02 \%$ & $36.27 \%$ & 27.92 & \\
\hline \multicolumn{2}{|c|}{ physiological state } & 403 & 10166 & $11.04 \%$ & $17.22 \%$ & 25.23 & \\
\hline \multicolumn{2}{|c|}{ laboratory technique } & 449 & 3161 & $12.30 \%$ & $5.35 \%$ & 7.04 & \\
\hline \multicolumn{2}{|c|}{ Total } & 3649 & 59043 & $100 \%$ & $100 \%$ & & \\
\hline
\end{tabular}

The first statistics depict the number and percentage of biological concepts and associated annotations, and the frequency of annotations per category. Besides individual categories, there are hierarchically structured annotation categories: the categories dna, rna and gene belong to the supercategory genetic information carrier; and the categories protein, enzyme and transcription factor are subcategories of protein. For these categories, the concept distribution of a category is then calculated by dividing the number of biological concepts assigned to the category per the total number of biological concepts assigned to its supercategory. Legend: The symbol "\#" stands for "number of" and the symbol "\%" stands for "percentage of". Frequencies are calculated as follows:

$$
\begin{aligned}
& \text { concept distribution }=\frac{\text { number of biological concepts in category }}{\text { total number of biological concepts in supercategory }} \\
& \text { annotation frequency }=\frac{\text { number of annotations in category }}{\text { number of biological concepts in category }}
\end{aligned}
$$

the expression of many genes. In addition, the low ratios of the categories protein, enzyme and transcriptional factor (below 5\%, $11 \%$ and $2 \%$, respectively), and subcategories $r n a$ and $d n a$ in the genetic information carrier supercategory (around 3\%) confirm that most of the discussion on cellular responses is centred on genes and compounds.

It is also important to notice that the category physiological state represents $11 \%$ of the biological concepts in the corpus and $17 \%$ of the annotations in the corpus, with a high frequency of annotation per concept (around 25 annotations). Additionally, in the laboratory technique category, encompassing biological concepts that establish a mechanistic link between genes, compounds and proteins, the ratio of biological concepts is about $12 \%$ but the ratio of annotations is less than half of this, with a frequency of 7 annotations per technique.

\section{Comparative analysis of full-text and abstract assignments}

Compared to full-texts, abstracts are less rich and complex text segments. Abstracts tend to mention only key

Table 5 General statistics about agreement rates and concept assignments for the two corpora

\begin{tabular}{ccccc}
\hline & \multicolumn{2}{c}{ Abstracts } & \multicolumn{2}{c}{ Full-texts } \\
\cline { 2 - 5 } & F-scores & Final number of biological concepts & F-scores & Final number of biological concepts \\
\hline$d n a$ & $30.77 \%$ & 25 & $13.22 \%$ & 126 \\
\hline rna & $81.82 \%$ & 32 & $59.69 \%$ & 119 \\
\hline gene & $87.84 \%$ & 73 & $91.78 \%$ & 1175 \\
\hline protein & $45.16 \%$ & 35 & $42.15 \%$ & 175 \\
\hline enzyme & $70.18 \%$ & 67 & $63.33 \%$ & 388 \\
\hline transcription factor & $20 \%$ & 17 & $28.13 \%$ & 47 \\
\hline compound & $83.09 \%$ & 188 & $63.90 \%$ & 767 \\
\hline biochemical reaction & $0 \%$ & $-\left(^{*}\right)$ & $0 \%$ & $-\left(^{*}\right)$ \\
\hline physiological state & $46.63 \%$ & 145 & $46.50 \%$ & 403 \\
\hline laboratory technique & $75.27 \%$ & 58 & $38.34 \%$ & 449 \\
\hline
\end{tabular}

The F-score columns refer to the F-score values achieved for the 130 documents after training and before post-processing; and the final number of biological concepts is calculated after post-processing.

$\left.{ }^{*}\right)$ This biological concept was not included in the final corpora. See the Post-processing sub-section for more details. 
biological concepts and the text is quite concise. So, we would expect that abstract annotation would be significantly less affected by differing levels of expertise.

To be able to draw a fair comparison, we have used only the 130 documents that are common to both corpora. We consider the improvement of the rates of agreement during the training period (which only covered 45 of the 130 documents) and the rate of agreement for the 130 documents, and the final distribution of biological concepts per semantic category (Table 5). During training, abstracts have a greater improvement of the rates for most categories, except for the subcategories of gene, i.e. $d n a$ and rna, and the categories compound and physiological state. This could be explained by the same problems found during training on full papers, where the assignment of transcription units or operons as gene is confused with $d n a$ (e.g. operon cyoABCDE), and the inclusion of compounds that are not participants in the metabolism, but are chemical compounds used in assays (e.g. EDTA, a chelating agent used for gel electrophoresis), were detected. These misclassifications would decrease the IAA measurements.

When constructing the corpora of 130 documents, the rates of agreement achieved for abstracts are significantly higher than those achieved for full-texts, except for gene ( $4 \%$ less) and transcription factor ( $8 \%$ less) assignments. For these biological concepts, one of the annotators assigned fewer terms, resulting in lower agreement rates. It was found that most terms were annotated as the supercategory (i.e., protein) by one of the annotators, while the other discriminated the functional role of those proteins (e.g. CRP and Lrp that DNA-binding transcriptional regulators). The same happened when annotating full-texts, but the proportion of these misclassified terms was lower, which contributed to a slightly increased F-score.

\section{Conclusions}

We have designed a schema and a set of guidelines in support of the semantic annotation of microbial cellular responses. We have produced a corpus of 130 freeaccess full-texts with a total of 59043 annotations, corresponding to 3649 unique biomedical concepts.

Through discrepancy analysis of the corpus, we have pin-pointed the most problematic issues for annotators, both in terms of terminological and background-related issues. Our results show that high levels of agreement (over 90\%) can only be achieved for the gene category. The average agreement rate for most of the other categories is around $50 \%$. The exceptions are the categories $d n a$ and biochemical reaction that have very low levels of agreement. So, it is interesting to notice that even amongst experienced researchers, annotation is still subjective and highly dependent on whether or not the researcher is familiar with the cellular processes under annotation. Specifically, such high familiarity is required in the annotation of different types of biological concepts (often sharing common names) that play distinct cellular roles depending on the context of the statement. This is considered a major concern for the purpose of reconstructing cellular models. The size of the model and its complexity may hide some mis-annotations (or misextractions) and lead to false biological interpretations.

This full-text corpus is suitable for use in the validation of the ability of information extraction tools in ambiguous contexts. Moreover, since the corpus encompasses a wide variety of biological concepts at the core of cellular responses, we believe that it may be a useful resource in the development of text mining tools supporting the reconstruction of cellular models. The corpus is freely accessible at http://sysbio.uminho.pt/ corpus_ecoli.

\section{Acknowledgements and funding}

This work is partly funded by SYSINBIO, an European Coordination and Support action (call FP7-KBBE-2007-1) in the field of model driven metabolic engineering, and the Portuguese FCT (Fundação para a Ciência e Tecnologia) funded MIT-Portugal Program in Bioengineering (MIT-Pt/BS-BB/ 0082/2008). The work of Rafael Carreira, Sónia Carneiro and Rui Pereira are supported by PhD grants from FCT (refs. SFRH/BD/66201/2009, SFRH/BD/ 22863/2005 and SFRH/BD/51111/2010, respectively).

\section{Author details}

${ }^{1}$ IBB - Institute for Biotechnology and Bioengineering, Centre of Biological Engineering, University of Minho, Campus de Gualtar, 4710-057 Braga PORTUGAL. ${ }^{2}$ Department of Informatics/CCTC, University of Minho, Campus de Gualtar, 4710-057 Braga - PORTUGAL.

\section{Authors' contributions}

All authors participated in the preparation of the manuscript. AL supervised all steps of the work. SC and RP performed the annotations, whilst ECF and IR prepared the annotation scheme and oriented training discussion, covering for key biological issues. RC and MR provided technical support during the training phase and were responsible for compiling corpus statistics and evaluating IAA. The analysis of annotation discrepancies derived from a joint effort. All authors read and approved the final manuscript.

Received: 18 May 2011 Accepted: 28 November 2011 Published: 28 November 2011

\section{References}

1. Harmston N, Filsell W, Stumpf MP: What the papers say: Text mining for genomics and systems biology. Hum Genomics 2010, 5:17-29.

2. Krallinger $M$, Leitner $F$, Valencia A: Analysis of biological processes and diseases using text mining approaches. Methods Mol Biol 2010, 593:341-382.

3. Kowald A, Schmeier S: Text mining for systems modeling. Methods Mol Biol 2011, 696:305-318.

4. Kemper B, Matsuzaki T, Matsuoka Y, Tsuruoka Y, Kitano H, Ananiadou S, Tsujii J: PathText: a text mining integrator for biological pathway visualizations. Bioinformatics 2010, 26:i374-i381.

5. Rodriguez-Penagos C, Salgado H, Martinez-Flores I, Collado-Vides J: Automatic reconstruction of a bacterial regulatory network using Natural Language Processing. BMC Bioinformatics 2007, 8.

6. Griffith OL, Montgomery SB, Bernier B, Chu B, Kasaian K, Aerts S, Mahony S, Sleumer MC, Bilenky M, Haeussler M, Griffith M, Gallo SM, Giardine B, Hooghe B, Van LP, Blanco E, Ticoll A, Lithwick S, Portales-Casamar E, Donaldson IJ, Robertson G, Wadelius C, De BP, Vlieghe D, Halfon MS, 
Wasserman W, Hardison R, Bergman CM, Jones SJ: ORegAnno: an openaccess community-driven resource for regulatory annotation. Nucleic Acids Res 2008, 36:D107-D113.

7. Heinen S, Thielen B, Schomburg D: KID-an algorithm for fast and efficient text mining used to automatically generate a database containing kinetic information of enzymes. BMC Bioinformatics 2010, 11:375.

8. Scheer M, Grote A, Chang A, Schomburg I, Munaretto C, Rother M, Sohngen C, Stelzer M, Thiele J, Schomburg D: BRENDA, the enzyme information system in 2011. Nucleic Acids Res 2011, 39:D670-D676.

9. Bult CJ, Kadin JA, Richardson JE, Blake JA, Eppig JT: The Mouse Genome Database: enhancements and updates. Nucleic Acids Res 2010, 38 : D586-D592.

10. Jensen $\sqcup$, Kuhn M, Stark M, Chaffron S, Creevey C, Muller J, Doerks T, Julien P, Roth A, Simonovic M, Bork P, von MC: STRING 8-a global view on proteins and their functional interactions in 630 organisms. Nucleic Acids Res 2009, 37:D412-D416.

11. Zaremba S, Ramos-Santacruz M, Hampton T, Shetty P, Fedorko J, Whitmore J, Greene JM, Perna NT, Glasner JD, Plunkett G, Shaker M, Pot D: Text-mining of PubMed abstracts by natural language processing to create a public knowledge base on molecular mechanisms of bacterial enteropathogens. BMC Bioinformatics 2009, 10:177.

12. Spasic I, Simeonidis E, Messiha HL, Paton NW, Kell DB: KiPar, a tool for systematic information retrieval regarding parameters for kinetic modelling of yeast metabolic pathways. Bioinformatics 2009, 25:1404-1411.

13. Lourenço A, Carreira R, Carneiro S, Maia P, Glez-Peña D, Fdez-Riverola F, Ferreira EC, Rocha I, Rocha M: @Note: A workbench for Biomedical Text Mining. Journal of Biomedical Informatics 2009.

14. Kano Y, Dobson P, Nakanishi M, Tsujii J, Ananiadou S: Text mining meets workflow: linking U-Compare with Taverna. Bioinformatics 2010, 26:2486-2487.

15. Krallinger M, Valencia A: BioCreative III, PPI Task. 2010 [http://www. biocreative.org/tasks/biocreative-iii/ppi/].

16. Leitner F, Mardis SA, Krallinger M, Cesareni G, Hirschman LA, Valencia A: An Overview of BioCreative II.5. IEEE/ACM Trans Comput Biol Bioinform 2010, 7:385-399

17. Hersh W, Bhupatiraju RT: TREC Genomics Track Overview. 2003, 14-23.

18. Hersh W, Bhupatiraju RT, Ross L, Johnson P, Cohen AM, Kraemer DF: TREC 2004 Genomics Track Overview. 2004, 13-31.

19. Kim JD, Ohta T, Pyysalo S, Kano Y, Tsujii J: Overview of BioNLP'09 shared task on event extraction. 2009, 1-9.

20. Kim JD, Ohta T, Tateisi Y, Tsujii J: GENIA corpus-semantically annotated corpus for bio-textmining. Bioinformatics 2003, 19(Suppl 1):i180-i182.

21. Pyysalo S, Ginter F, Heimonen J, Bjorne J, Boberg J, Jarvinen J, Salakoski T: Biolnfer: a corpus for information extraction in the biomedical domain. BMC Bioinformatics 2007, 8.

22. Pyysalo S, Airola A, Heimonen J, Bjorne J, Ginter F, Salakoski T: Comparative analysis of five protein-protein interaction corpora. BMC Bioinformatics 2008, 9.

23. Tanabe L, Xie N, Thom LH, Matten W, Wilbur WJ: GENETAG: a tagged corpus for gene/protein named entity recognition. BMC Bioinformatics 2005, 6.

24. Thompson P, labal SA, McNaught J, Ananiadou S: Construction of an annotated corpus to support biomedical information extraction. BMC Bioinformatics 2009, 10.

25. Leitner F, Krallinger M, Cesareni G, Valencia A: The FEBS Letters SDA corpus: a collection of protein interaction articles with high quality annotations for the BioCreative 11.5 online challenge and the text mining community. FEBS Lett 2010, 584:4129-4130.

26. Cohen KB, Johnson HL, Verspoor K, Roeder C, Hunter LE: The structural and content aspects of abstracts versus bodies of full text journal articles are different. BMC Bioinformatics 2010, 11:492.

27. Settles B: ABNER: an open source tool for automatically tagging genes, proteins and other entity names in text. Bioinformatics 2005 , 21:3191-3192.

28. Corbett $P$, Batchelor $C$, Teufel S: Annotation of chemical named entities. BioNLP 2007: Biological, translational, and clinical language processing 2007, 57-64.

29. Mika S, Rost B: NLProt: extracting protein names and sequences from papers. Nucleic Acids Res 2004, 32:W634-W637.
30. Tsuruoka Y, Tateishi Y, Kim JD, Ohta T, McNaught J, Ananiadou S, Tsujii J: Developing a robust part-of-speech tagger for biomedical text. Advances in Informatics, Proceedings 2005, 3746:382-392.

31. Leaman R, Gonzalez G: BANNER: an executable survey of advances in biomedical named entity recognition. Pac Symp Biocomput 2008, 652-663.

32. McIntosh T, Curran JR: Challenges for automatically extracting molecular interactions from full-text articles. BMC Bioinformatics 2009, 10:311.

33. Wang $H$, Huang $M$, Zhu $X$ : Extract interaction detection methods from the biological literature. BMC Bioinformatics 2009, 10(Suppl 1):S55.

34. Gerner M, Nenadic G, Bergman CM: LINNAEUS: a species name identification system for biomedical literature. BMC Bioinformatics 2010, 11:85.

35. Kim JD, Ohta T, Tsujii J: Corpus annotation for mining biomedical events from literature. BMC Bioinformatics 2008, 9.

36. Rajagopala SV, Goll J, Gowda ND, Sunil KC, Titz B, Mukherjee A, Mary SS, Raviswaran N, Poojari CS, Ramachandra S, Shtivelband S, Blazie SM, Hofmann J, Uetz P: MPI-LIT: a literature-curated dataset of microbial binary protein-protein interactions. Bioinformatics 2008, 24:2622-2627.

37. Oda K, Kim JD, Ohta T, Okanohara D, Matsuzaki T, Tateisi Y, Tsujii J: New challenges for text mining: mapping between text and manually curated pathways. BMC Bioinformatics 2008, 9.

38. Rocha I, Forster J, Nielsen J: Design and application of genome-scale reconstructed metabolic models. In Microbial Gene Essentiality: Protocols and Bioinformatics Edited by: Osterman AL, Gerdes S 2008, 416:409-431, In series: Methods in Molecular Biology.

39. Pinto JP, Dias O, Lourenço A, Carneiro S, Ferreira EC, Rocha I, Rocha M: Data Integration Issues in the Reconstruction of the Genome-Scale Metabolic Model of Zymomonas Mobillis. Advances in Soft Computing 2009, 92-101.

40. Mendes R, Lourenço A, Carneiro S, Ferreira EC, Rocha I, Rocha M: A Framework for the Integrated Analysis of Metabolic and Regulatory Networks. The 8th IEEE International Conference on Biolnformatics and BioEngineering (IEEE BIBE 2008) 2008.

41. Carneiro S, Rocha I, Ferreira EC: Application of a genome-scale metabolic model to the inference of nutritional requirements and metabolic bottlenecks during recombinant protein production in Escherichia coli. Microb Cell Fact 2006, 5(Suppl 1).

42. Carneiro S, Amaral AL, Veloso AC, Dias T, Peres AM, Ferreira EC, Rocha I: Assessment of physiological conditions in E. coli fermentations by epifluorescent microscopy and image analysis. Biotechnol Prog 2009 25:882-891.

43. Carneiro S, Villas-Bôas S, Rocha I, Ferreira EC: Applying a metabolic footprinting approach to characterize the impact of the recombinant protein production in Escherichia coli. Advances in Soft Computing edition 2010, 193-200

44. Lourenço A, Carreira R, Glez-Peña D, Méndez JR, Carneiro S, Rocha LM Díaz F, Ferreira EC, Rocha I, Fdez-Riverola F, Rocha M: BioDR: Semantic indexing networks for biomedical document retrieval. Expert Systems with Applications 2010, 37:3444-3453

45. Keseler IM, Collado-Vides J, Santos-Zavaleta A, Peralta-Gil M, Gama-Castro S, Muniz-Rascado L, Bonavides-Martinez C, Paley S, Krummenacker M, Altman T, Kaipa P, Spaulding A, Pacheco J, Latendresse M, Fulcher C, Sarker M, Shearer AG, Mackie A, Paulsen I, Gunsalus RP, Karp PD: EcoCyc: a comprehensive database of Escherichia coli biology. Nucleic Acids Res 2010.

46. Chatr-aryamontri A, Kerrien S, Khadake J, Orchard S, Ceol A, Licata L, Castagnoli L, Costa S, Derow C, Huntley R, Aranda B, Leroy C, Thorneycroft D, Apweiler R, Cesareni G, Hermjakob H: MINT and IntAct contribute to the Second BioCreative challenge: serving the text-mining community with high quality molecular interaction data. Genome Biol 2008, 9(Suppl 2):S5.

47. Lourenço A, Carreira R, Carneiro S, Maia P, Glez-Peña D, Fdez-Riverola F, Ferreira EC, Rocha I, Rocha M: @Note: A workbench for Biomedical Text Mining. Journal of Biomedical Informatics 2009.

48. Brants T: Inter-annotator agreement for a German newspaper corpus. In the Second International Conference on Lanquage Resources and Evaluation (LREC-2000) 2000, 1435-1439.

doi:10.1186/1471-2105-12-460

Cite this article as: Carreira et al: Semantic annotation of biological concepts interplaying microbial cellular responses. BMC Bioinformatics 2011 12:460. 